\title{
Experimental investigation of burning characteristics of porous combustible soaked in liquid oxidizer
}

\author{
Daiki MATSUGI*, Yosuke HASEBE*, Takuya YAMAZAKI*, Tsuneyoshi MATSUOKA* \\ and Yuji NAKAMURA* \\ *Department of Mechanical Engineering, Toyohashi University of Technology \\ 1-1 Hibarigaoka, Tempaku, Toyohashi, Aichi 441-8580, Japan \\ E-mail: yuji@me.tut.ac.jp
}

Received: 28 April 2021; Revised: 2 July 2021; Accepted: 30 July 2021

\begin{abstract}
Experimental investigations of burning characteristics of a tested specimen consisting of a polyethylene foam soaked in $100 \mathrm{wt} \%$ hydrogen peroxide are made. All experiments are carried out in a large volume chamber, which newly introduced in our previous work at an initial pressure range from $p=0.1$ to $p=0.35 \mathrm{MPa}$ in absolute with various fuel porosity range from $\varepsilon=0.6$ to $\varepsilon=0.9$, which corresponds to global equivalence ratios from $\phi$ $=0.51$ to $\phi=3.8$. Temperature measurements using an R-type thermocouple embedded into the specimen are then conducted to investigate thermal structure (e.g., profiles of temperature and temperature gradients and burning surface temperature) of the burning specimen for deep understandings of the burning process. Following forced ignition at top surface of the specimen, steady successive-burning process is successfully observed for all conditions studied in this work. Burning rates (a rate at which the top surface moves downward) are measured by carefully tracking the top surface of the specimen by adopting an image processing software. Findings show that overall burning rates at the rate from 1 to $3.2 \mathrm{~mm} / \mathrm{s}$ are obtained and influenced by pressure and the fuel porosity. Additionally, results of the direct temperature measurements reveal that the temperature gradient in the gas-phase layer near the top surface (burning surface) of the specimen increases as pressure increases, resulting in an increase in the overall burning rate. The top surface temperature and its pressure dependency, and a global activation energy at the top surface temperature of the specimen are experimentally measured accordingly.
\end{abstract}

Keywords : Porous combustible, Liquid oxidizer, Premixed combustion, Burning rate, Burning surface temperature, Global activation energy

\section{Introduction}

Composite solid propellants based on ammonium perchlorate (called AP-based propellant) have been widely used for launcher boosters and strategical and tactical missiles for several decades (e.g., Shioya et al., 2014; Fitzgerald and Brewster, 2004). The AP-based propellants have been drawing attractive advantages, such as structural simplicity, higher reliability in operation than liquid propellants, and a relatively simple manufacturing process (Aziz et al., 2015; Zhu et al., 2017). However, it has been considered that the AP-based propellants have given negative impacts on the environment and our health, for instance, ozone layer depletion, acid rain, and atmospheric contamination. (Chaturvedi and Dave, 2013; Aggarwal et al., 2015). According to the European and international agendas, green propellants (namely, environmentally friendly propellants to minimize those negative impacts) have become a top priority for sustainable developments and future use (Wingborg and Calabro, 2017). Furthermore, ammonium perchlorate behaves as an explosive (Essel et al., 2019), so that it is carefully handled to ensure our safety from explosion and its toxicity (Aggarwal et al., 2015). For aforementioned reasons, many research groups have been exploring an alternative green propellant, such as hybrid rocket propellants and AP-free solid propellants, to replace into the AP-based propellant (e.g., Nagata et al., 1997; Sabourin et al., 2008; Sundaram et al., 2014; Sinditskii and Egorshev, 2008; Abd-Elghany et al., 2018). Nevertheless, upgrading these green propellants is no doubt challenging.

One of the promising candidates for the green propellant is to employ a well-mixing propellant consisting of a porous 
combustible (polymeric material or metal particles group) well-soaked in liquid oxidizer (e.g., Nagata et al., 1997; Sabourin et al., 2008; Sundaram et al., 2014), featuring a large specific surface-volume ratio (S/V). The heat and mass transfer rate shall be, thus, intensified by means of the propellant. Based on this concept, Nagata et al. (1997) carried out a series of experiments in a large volume chamber and in a nitrogen environment with a $50 \mathrm{~mm}$-long specimen consisting of fibrous polyamide (the porous combustible) soaked in liquid oxygen (LOX) with a controlled pressure environment (Nagata et al., 1997). They measured burning rates by signals from three thermocouples (TCs) embedded into the specimen and obtained a maximum burning rate at the rate of $20 \mathrm{~mm} / \mathrm{s}$, which corresponds to about $24 \mathrm{~kg} /\left(\mathrm{m}^{2} \cdot \mathrm{s}\right)$ and two-times higher than that of the AP-based propellants in terms of total mass flux (Sutton and Biblarz, 2017). This fact clearly proved that the unique idea of the well-mixing propellant sounds attractive.

In the previous work by Nagata et al. (1997), although the high burning rates were successfully achieved, large data scattering of the burning rates was often observed at random pressure environments even in a standard pressure environment $(0.1 \mathrm{MPa})$. In addition, the experimental data was limited to only the signals from the three TCs embedded, allowing them only to measure just an "average" burning rate. Probably one of the reasons for the large data scattering is attributed to this method. Furthermore, no visualization of the burning event was presented. This may be because the use of LOX would make it difficult to observe the burning event directly and control the tested environment; very low temperature in the whole experimental setup must be maintained to prevent LOX from vaporizing before experiments. Therefore, few fundamental burning characteristics of this specimen have been given so far since 1997.

For upgrading this unique idea, it is necessary to gather the fundamental knowledges of burning characteristics of the well-mixing specimen, such as pressure dependency and fuel property on whether steady burning is achieved or not, on burning behavior, the burning rate, and thermal structure that have not been presented enough. Just recently, we attempted to re-design of the experimental setup and the tested method, which enables us to examine those burning characteristics (Matsugi et al., 2021). The tested specimen was replaced into a polyethylene foam (PE foam) as the porous combustible and $80 \mathrm{wt} \%$ hydrogen peroxide as the liquid oxidizer for ease to handle and control the tested environment at room temperature. Following the replaced specimen, we newly introduced an experimental setup and a method which enable us to observe the burning event directly and measure the burning rates. This attempt successfully achieved the steady burning event. Based on the observation result, theoretical model to describe during the burning event was firstly developed.

In this work, as referred to our previous work (Matsugi et al., 2021), comprehensive experimental studies to investigate the fundamental burning characteristics of this specimen are made at an initial pressure range from $p=0.1$ to $p=0.35 \mathrm{MPa}$ in absolute under fuel porosity range from $\varepsilon=0.6$ to $\varepsilon=0.9$. Direct temperature measurements using an $\mathrm{R}-$ type thermocouple are made in order to examine thermal structure (e.g., profiles of temperature, temperature gradient and burning surface temperature) of the burning specimen for deep understanding of this burning process and a global activation energy of the specimen by taking advantages of the measured thermal status and its pressure response.

\section{Experiment}

\subsection{Experimental setup and tested specimen}

Figure 1 (A) and (B) show schematic diagrams of the whole experimental setup and an enlarged view of the tested specimen used in this work, respectively. All experiments are carried out in a large volume chamber $(300 \mathrm{~mm} \times 300 \mathrm{~mm}$ $\times 400 \mathrm{~mm}$ ) which is equipped with a pair of quartz windows to allow direct observation during the entire burning event. The tested specimen is composed of the polyethylene (PE) foam (Sakai Chemical Industry Co., Ltd.; 99 \% PE composition, $185 \mathrm{~kg} / \mathrm{m}^{3}$ apparent density) sized $18 \mathrm{~mm}$ in diameter and $60 \mathrm{~mm}$ in height, and it is fully soaked in 100 wt $\% \mathrm{H}_{2} \mathrm{O}_{2}$ (Mitsubishi Gas Chemical Company, Inc.; $60 \mathrm{wt} \% \mathrm{H}_{2} \mathrm{O}_{2}$ concentration from an original product) as the liquid oxidizer. Please note that the concentration procedure was upgraded from our previous work (Matsugi et al., 2021) at which $80 \mathrm{wt} \% \mathrm{H}_{2} \mathrm{O}_{2}$ is achieved. The tested specimen is then inserted into the vertically oriented transparent quartz tube for good visibility, which measures $18 \mathrm{~mm}$ in inner diameter, $50 \mathrm{~mm}$ in height and is closed off at one end of the quartz tube. As shown in Fig. 1 (B), about $10 \mathrm{~mm}$ of the PE foam is placed over the top edge of the quartz tube for ease of forced ignition. In the temperature measurement trials, the R-type thermocouple (TC) (The Nilaco Cooperation, $0.1 \mathrm{~mm}$ in diameter and about $0.2 \mathrm{~mm}$ of junction size in diameter) is embedded into the center of the specimen in radius direction at the distance of $25 \mathrm{~mm}$ below from the top edge with an inclined angle of about $30^{\circ}$ as shown in the figure. The TC is tensioned moderately not to be dragged by any negligible force, such as viscosity of melted PE foam, from the original 
position during the entire burning event. In addition, it is reasonable for the inclination of the TC to prevent the TC from being dragged due to the force because the right side of the TC (not junction) remains in solid phase while the TC's junction passes through the top surface of the specimen and the flame. With this respect, the TC is fixed at the original location during the burning event. Forced ignition is then induced by a U-shaped nickel-chrome wire (ignitor) that measures $0.8 \mathrm{~mm}$ in diameter. Once the specimen is set in the chamber, and the U-shaped ignitor is attached to the top surface of the specimen, pressurization with nitrogen gas is initiated. Only for the experiment at $p=0.1 \mathrm{MPa}$ in absolute, the nitrogen line and the purge line open during its experiment to allow us to supply nitrogen gas into the chamber to make a nitrogen environment. Except for $p=0.1 \mathrm{MPa}$, the purge line is closed off to make high pressure environment, and the pressurization is done by manual operation. In this work, since the pressurization with nitrogen gas is started from when air (21\% oxygen concentration in vol.) is contained in the chamber before the pressurization, for all the cases, it is considered that the inside the chamber is not fully filled with pure nitrogen. Pressure inside the chamber during entire the experiment is then measured by a pressure sensor (Valcom Co., LTD, VPRT (F), rated capacity: $1 \mathrm{MPa}$ ) which is connected to a wall of the chamber. Temporal signals from the pressure sensor and the TC are recorded by a data logger (GRAPHTEC, midi LOGGER GL900) at a $100 \mathrm{~Hz}$ sampling rate. After completing pressurization up to a desired value, $210 \mathrm{~W}$ power is applied to the ignitor for 5-10 s to achieve successful ignition. The ignitor is then removed from the top of the specimen to prevent the burning event from being disturbed. The whole burning behavior is recorded by a digital video camera (Canon, iVIS HF G20, shutter speed: 1/250, gain: $18 \mathrm{~dB}$, F-number: 2.8) through the view window attached to the chamber. The moving burning surface is traced using an imaging software ImageJ. The experiments are conducted at an initial pressure range from $p=0.1 \mathrm{MPa}$ to $p=0.35 \mathrm{MPa}$ in absolute and the fuel porosity range from 0.6 to 0.9. As a typical example of the pressure during the entire experiment, Fig. 1 (C) shows overall time history of the pressure inside the chamber when the averaged pressure is set as $p=0.22 \mathrm{MPa}$ in absolute. An initial time when we confirm the ignition at the top surface is adjusted to $t=0 \mathrm{~s}$. Four periods are then shown; a period while the ignitor is turned on and off, a period during the burning event, and a period after burning event, and a period during the purge event, respectively. An averaged pressure at the two points of the initial pressure $P_{s t}$ (when we confirm the ignition) and the final pressure $P_{\text {end }}$ (when the burning event finishes (flame quenches)) is employed in this work (see Fig. 1 (C)). We confirmed that all the experiments are conducted under almost the constant pressure environment as denoted on the figure (C), thus granting quasi-steady burning conditions. The reason for the slight fluctuation of the pressure in the burning time is due to the manual operation to achieve the desired pressure. After confirming that the burning event is completed, the purge line opens, and burned gas is exhausted outside the chamber.
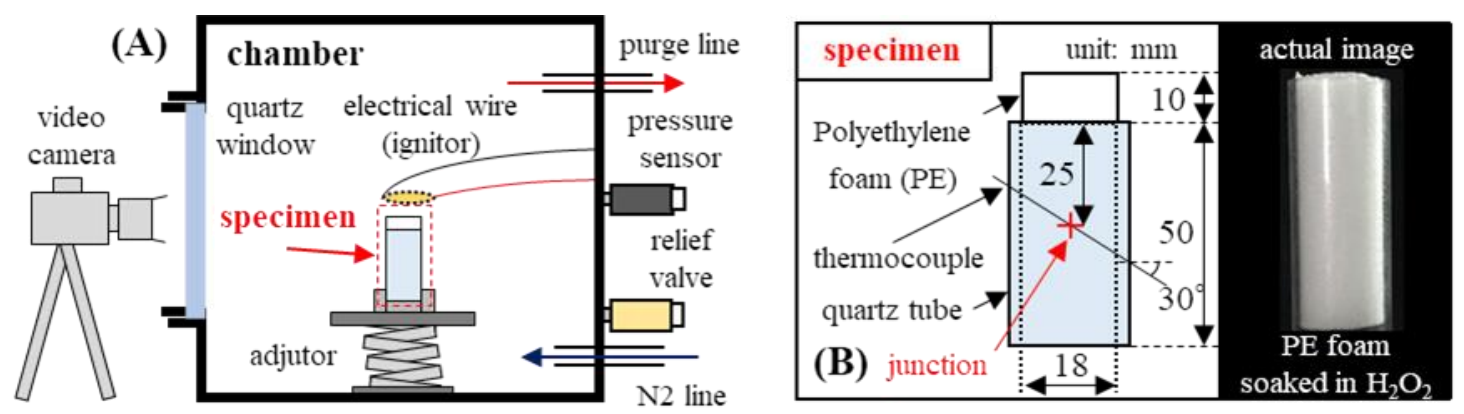

(C)

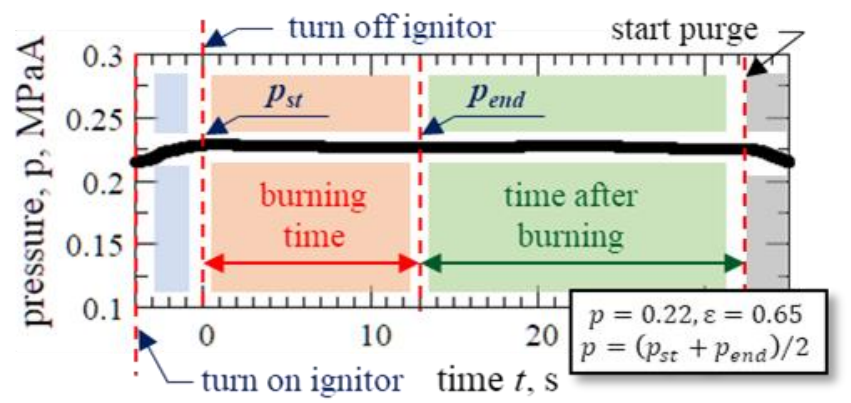

Fig. 1 Schematic diagram of (A) a whole experimental setup and (B) an enlarge view of the specimen and (C) typical time history of pressure ( $p=0.22 \mathrm{MPa}$ ) inside the chamber measured by the pressure sensor 


\subsection{Definition of global equivalence ratio depending on fuel porosity}

The fuel porosity of the PE foam is one of the fuel properties, and it is considered as affecting the overall burning characteristics. Definition of the fuel porosity is given by the following relation.

$$
\varepsilon=\frac{V_{L}}{V}=\frac{\frac{m_{L}}{\rho_{L}}}{\frac{m_{L}}{\rho_{L}}+\frac{m_{S}}{\rho_{S}}}
$$

where $\varepsilon[-]$ is the fuel porosity of the PE foam, $V_{L}\left[\mathrm{~m}^{3}\right]$ is the total pore volume in the PE foam, $V\left[\mathrm{~m}^{3}\right]$ is the total volume of the $\mathrm{H}_{2} \mathrm{O}_{2}$-soaked PE foam, $m_{L}[\mathrm{~kg}]$ is the initial mass of hydrogen peroxide contained in the PE foam, $m_{S}[\mathrm{~kg}]$ is the initial mass of the PE foam, $\rho_{s}\left[\mathrm{~kg} / \mathrm{m}^{3}\right]$ is the density of the PE foam, and $\rho_{L}\left[\mathrm{~kg} / \mathrm{m}^{3}\right]$ is the density of the hydrogen peroxide, respectively. The fuel porosity of the PE foam can be adjusted by making each mass $\left(m_{L}\right.$ and $\left.m_{S}\right)$ control from the original product (PE foam) by uniformly drilling along vertical direction with a very thin drill (0.1 mm in diameter). Each mass $\left(m_{L}\right.$ and $\left.m_{S}\right)$ is then measured by electric balance (Shimadzu Corporation, UW820S), and the fuel porosity can be determined accordingly. We made our best to fully soak hydrogen peroxide into the pores of the PE foam for every experiment. In case that $\mathrm{H}_{2} \mathrm{O}_{2}$ is completely soaked in the pores, and assuming that the steady burning process is achieved, an oxidizer-to-fuel mass ratio (namely, $O / F$ ) is introduced as a following relation using the fuel porosity $(\varepsilon)$ or each mass $\left(m_{L}\right.$ and $\left.m_{S}\right)$.

$$
\left(\frac{O}{F}\right)_{e x}=\frac{\varepsilon \rho_{L}}{(1-\varepsilon) \rho_{S}}=\frac{m_{L}}{m_{S}}
$$

Thus, global equivalence ratio $(\phi)$ is given as follows with the fuel porosity expression.

$$
\phi=\frac{\left(\frac{O}{F}\right)_{t h}}{\left(\frac{O}{F}\right)_{e x}}=\frac{\left(\frac{O}{F}\right)_{t h}}{\frac{\varepsilon \rho_{L}}{(1-\varepsilon) \rho_{S}}}
$$

Assuming that one-step overall reaction (Fuel $+v$ Oxidizer $\rightarrow(1+v)$ Product, $v$ is the stoichiometric coefficient based on a mass) is adopted, and pyrolysis gas of the PE foam mainly consists of ethylene $\left(\mathrm{C}_{2} \mathrm{H}_{4}\right)$, a stoichiometric oxidizer-to-fuel mass ratio $(O / F)_{t h}$ shall be 7.29 as calculated from the following overall chemical reaction.

$$
\mathrm{C}_{2} \mathrm{H}_{4}+6 \mathrm{H}_{2} \mathrm{O}_{2}=2 \mathrm{CO}_{2}+8 \mathrm{H}_{2} \mathrm{O}
$$

By giving the densities $\left(\rho_{L} ; 1450 \mathrm{~kg} / \mathrm{m}^{3}\right.$ and $\left.\rho_{S} ; 920 \mathrm{~kg} / \mathrm{m}^{3}\right)$, the relation between the global equivalence ratio and the fuel porosity is given in Fig. 2, and the fuel porosity for the stoichiometric condition is determined as $\varepsilon=0.82$.

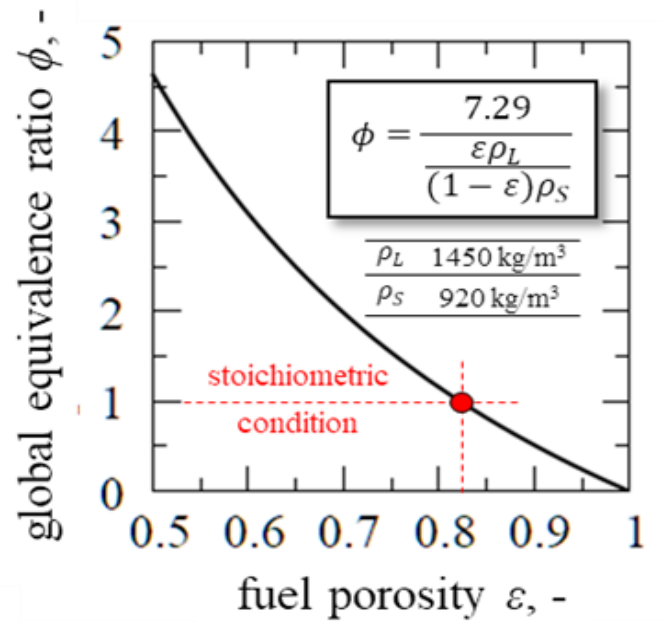

Fig. 2 Relation of global equivalence ratio $(\phi)$ as a function of fuel porosity ( $\varepsilon$ ) determined by Eq. (3) 


\section{Experimental results and discussion}

\subsection{Effect of pressure on subsequential flame shapes and location of surface flame}

Figure 3 shows the effect of pressure (A) $p=0.1 \mathrm{MPa}$, (B) $p=0.22 \mathrm{MPa}$, and (C) $p=0.29 \mathrm{MPa}$ with almost the constant fuel porosity $\varepsilon=0.65$ on the typical sequential flame shapes during the burning event. The origin of the $x$, coordinate $\left(x^{\prime}=0\right)$ is fitted to the top edge of the quartz tube. The global equivalence ratios that correspond to $\varepsilon=0.65$ are $\phi=2.5$, calculated based on Eq. (3). Therefore, combustion for all the cases here is taken place under the fuel-rich condition. After the successful ignition at the top surface of the specimen, it is confirmed that the top surface of the specimen moves downward for all the cases. In addition, it is identified that two distinctive flames are established. One is an elongated diffusion flame (an outer flame), the other is a surface flame (a brighter flame) around the exposed surface, as illustrated in Fig. 3 (D). For this type of combustion, thermal pyrolysis (gasification) of the PE foam gradually occurs at the location beneath the top surface, and vaporization of the hydrogen peroxide is expected to occur deeper than the location where the thermal pyrolysis is initiated. Taking into the account this fact, the premixed-gas mixture (mixture of the gasified fuel and the vaporized oxidizer) is formed inside the PE foam and will be supplied into the flame, establishing the premixed-like surface flame near the top surface. The outer flame is then established because the premixed-gas mixture which does not consume at the surface flame reacts again with the oxygen slightly containing in the ambient gas. Hence, if nitrogen gas is fully filled in the chamber, it is expected that the outer flame does not appear. Additionally, it is also identified that the luminosity of the surface flame increases as pressure increases. As pressure increases, in general, adiabatic flame temperature and soot formation are increased (McCrain and Roberts, 2005). These may contribute to the change in the flame brightness.

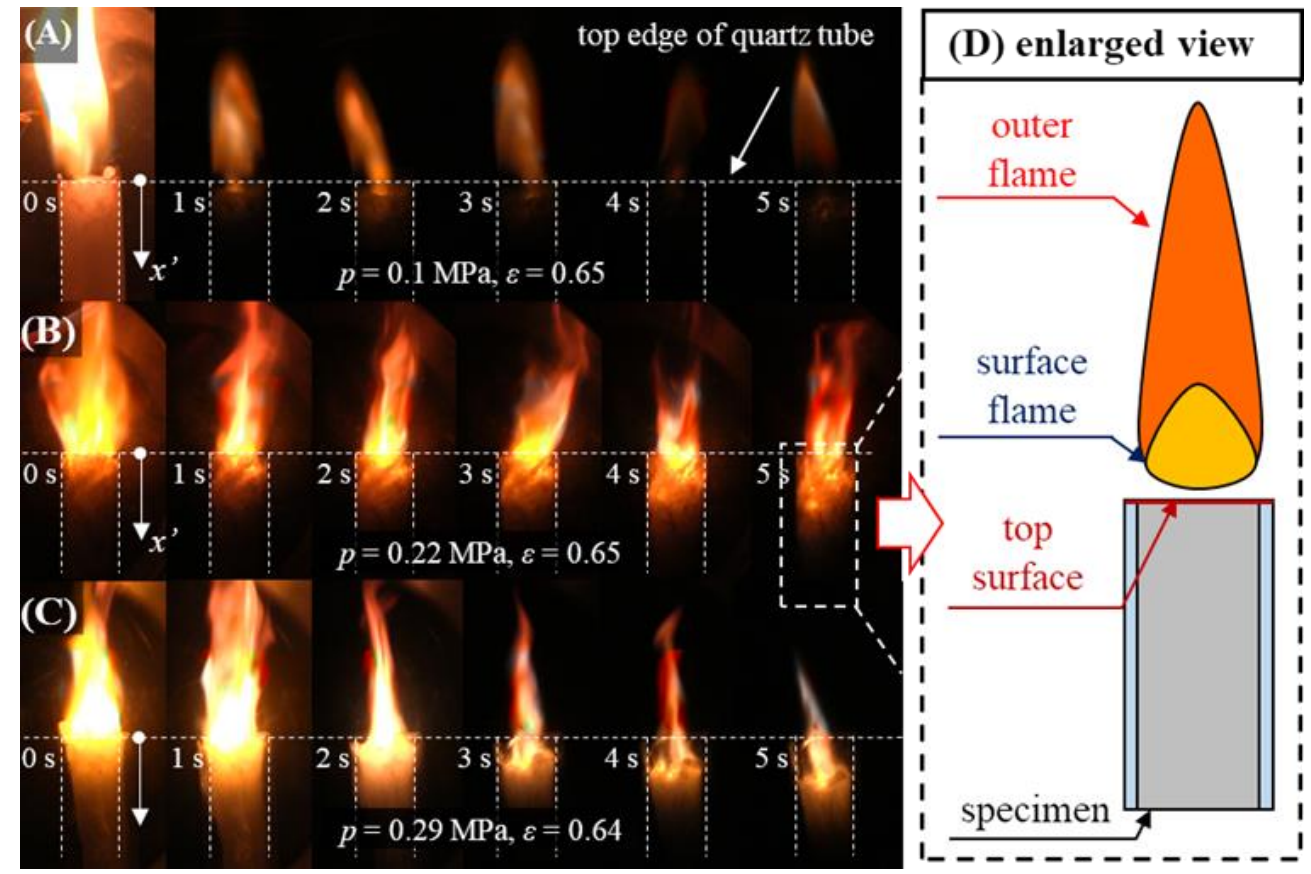

Fig. 3 Typical sequential flame shapes after forced ignition at top surface of specimen with $\varepsilon=0.65$ of fuel porosity at (A) $p=0.1 \mathrm{MPa}$, (B) $p=0.22 \mathrm{MPa}$, and (C) $p=0.29 \mathrm{MPa}$ in absolute, and (D) schematic diagram of surface flame and outer flame

In order to quantitatively evaluate the burning rates for the conditions of (A) $p=0.1 \mathrm{MPa},(\mathrm{B}) p=0.22 \mathrm{MPa}$, and $(\mathrm{C})$ $p=0.29 \mathrm{MPa}$, respectively, time histories of locations of the surface flame attached to the top surface are plotted in Fig. 4 by adopting the image processing software. In the figure, lines obtained from the least squared method for each condition are also shown. The gradient of the lines shows the linear burning rate (called "overall burning rate" hereafter). It is obvious that the surface flame moves downward with reasonably constant rates of (A) $1.6 \mathrm{~mm} / \mathrm{s}\left(R^{2}: 0.9655\right)$, (B) $2.4 \mathrm{~mm} / \mathrm{s}\left(R^{2}: 0.9897\right)$, and (C) $2.9 \mathrm{~mm} / \mathrm{s}\left(R^{2}: 0.9762\right)$, respectively, with satisfactory $R^{2}$ (coefficient of determination) values. For the sake of convenience, the burning event when obtaining overall burning rates with more than 0.96 in the $R^{2}$ value are defined as "steady burning" in this work. 

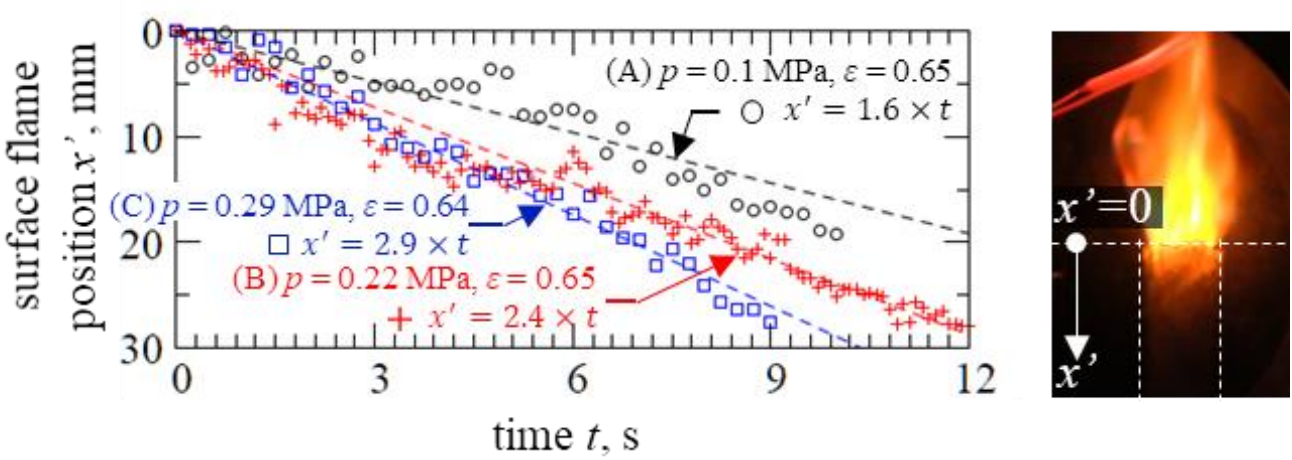

Fig. 4 Time history of locations of surface flame obtained from image processing software under (A) $p=0.1 \mathrm{MPa},(\mathrm{B}) p=$ $0.22 \mathrm{MPa}$, and (C) $p=0.29 \mathrm{MPa}$ in absolute with almost the constant fuel porosity $\varepsilon=0.65$

\subsection{Effect of fuel porosity on subsequential flame shapes and location of surface flame}

Figure 5 shows the effect of the fuel porosity $(\mathrm{A}) \varepsilon=0.65$, (B) $\varepsilon=0.72$, and $(\mathrm{C}) \varepsilon=0.80$ on the burning behavior during the burning event at standard pressure ( $p=0.1 \mathrm{MPa}$ in absolute), respectively. The global equivalence ratios that correspond to those fuel porosities are (A) $\phi=2.5(\varepsilon=0.65),(\mathrm{B}) \phi=1.8(\varepsilon=0.72)$, and $(\mathrm{C}) \phi=1.2(\varepsilon=0.80)$, respectively. Therefore, combustion in (A) and (B) is taken place under fuel-rich condition, and combustion in (C) is taken place under almost the stoichiometric condition. For all the cases, the outer flame and the surface flame as described in the previous section are identified. As mentioned, it is considered that inside the chamber is not completely full of nitrogen gas, so that the outer flames are pronounced. In comparison with all the conditions, as the fuel porosity approaches to the stoichiometric condition $(\varepsilon=0.8)$, the luminosity of the two flames is increased. This is attributed to an increase in the flame temperature when the fuel porosity approaches to the stoichiometric condition. Moreover, it is seen that the outer flame elongates when the fuel porosity approaches to the stoichiometric conditions. Especially for when the fuel porosity approaches to the stoichiometric condition from such a fuel-rich condition, the regression of the specimen is promoted, and the amount of the (fuel rich) premixed-gas mixture which does not consume at the surface flame is increased. This leads to that the outer flame elongates.

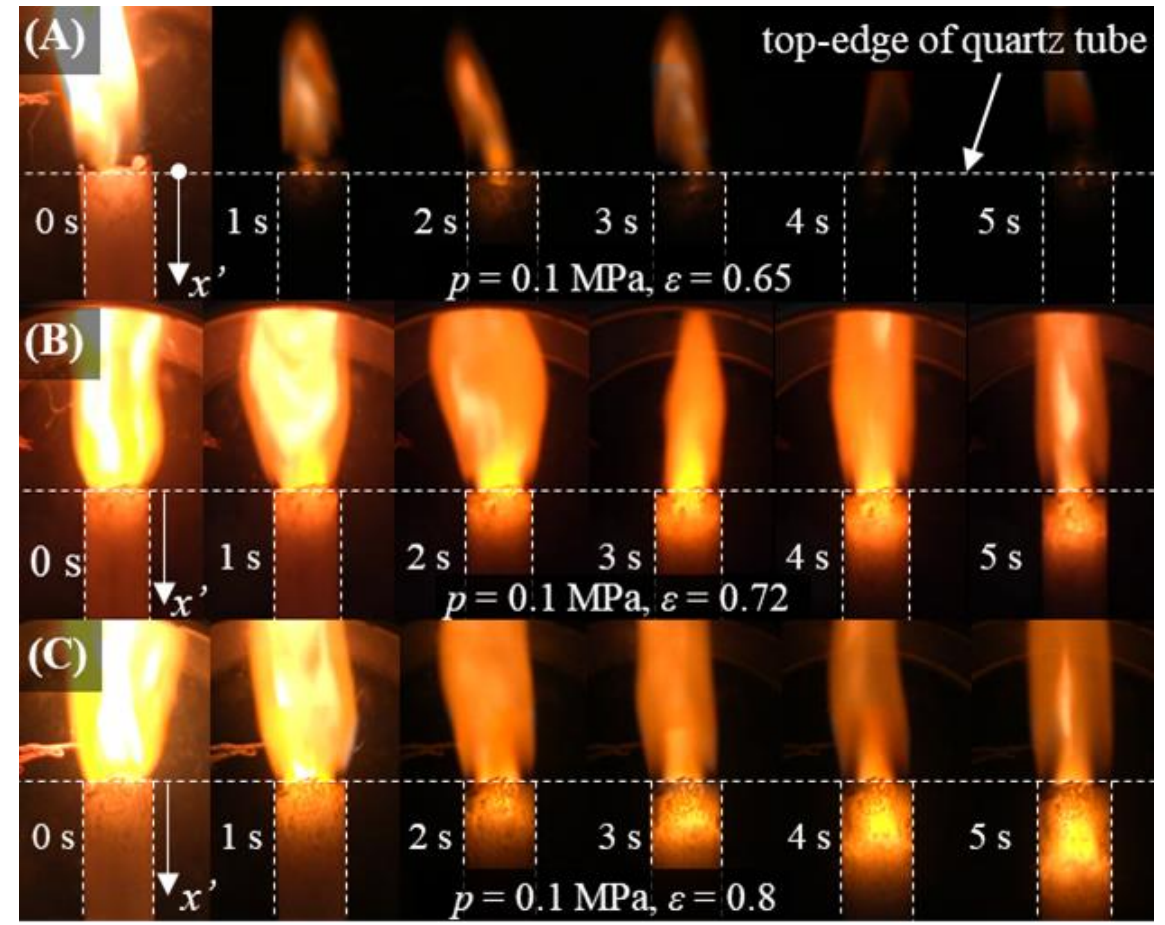

Fig. 5 Typical sequential flame shape after forced ignition at top surface of specimen around $p=0.1$ MPa in nitrogen environment with fuel porosities (A) $\varepsilon=0.65$, (B) $\varepsilon=0.72$, and (C) $\varepsilon=0.8$. 
Again, the time histories of the locations of the surface flame are plotted in Fig. 6 for $(\mathrm{A}) \varepsilon=0.65$, (B) $\varepsilon=0.72$, and (C) $\varepsilon=0.8$ at $p=0.1 \mathrm{MPa}$ in absolute, together with the lines obtained from the least squared method. It is obvious that the surface flame moves downward with reasonably constant at the rate of $(\mathrm{A}) 1.6 \mathrm{~mm} / \mathrm{s}\left(R^{2}: 0.9655\right),(\mathrm{B}) 1.8 \mathrm{~mm} / \mathrm{s}\left(R^{2}\right.$ : $0.9932)$, and (C) $2.7 \mathrm{~mm} / \mathrm{s}\left(R^{2}: 0.9950\right)$, respectively with satisfactory $R^{2}$ value as well. By these facts, we may safely say that the present specimen can achieve steady burning event experimentally in the conditions studied. On the other hand, it is seen that slight fluctuations of the locations of the flame surface are plotted in both the figures (Fig. 4 and Fig. 6). This is attributed to formation of molten PE adhered to the inner wall of the quartz tube during the burning event. Although this makes it difficult to visualize the exact location of the surface flame, it does not hinder the experimental computation of the overall burning rate in this system.

In all the experimental conditions, it seems that the surface flames are attached to the top surface of the specimen all the time. At least, we did not observe phenomenon such as flame spreading mode under studied conditions. The direct observation reveals that the burning event occurs at which the top surface moves downward constantly and indicating that combustion in this specimen occurs as an end burning mode.

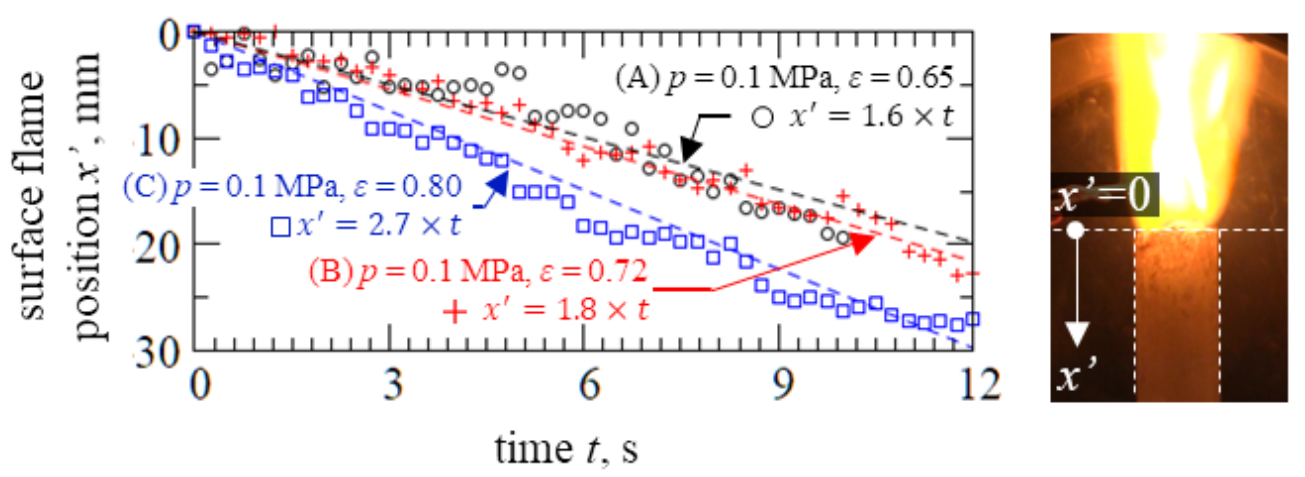

Fig. 6 Time history of location of surface flame obtained from image processing software at $p=0.1 \mathrm{MPa}$ in absolute with (A) $\varepsilon=0.65$, (B) $\varepsilon=0.72$, and (C) $\varepsilon=0.8$, together with lines obtained from least squared method

\subsection{Effect of fuel porosity and pressure on overall burning rate}

In this section, we would like to summarize the overall burning rates obtained for various pressure and the fuel porosity. Fig. 7 (A) shows the overall burning rate versus the fuel porosity for $p=0.1 \mathrm{MPa}, 0.15 \mathrm{MPa}, 0.2 \mathrm{MPa}, 0.25$ $\mathrm{MPa}$, and 0.3 MPa in absolute. The global equivalence ratios corresponding to the fuel porosity are shown on the second horizontal axis. Four-runs experiments for each porosity and pressure are carried out for repeatability. The error bars shown on the figure are calculated by $95 \%$ confidence interval. It is clearly seen that the overall burning rate varies depending on the fuel porosity and pressure. The repeatability (the error bars) is found to be less than $1 \mathrm{~mm} / \mathrm{s}$ for all the cases. In this work, the overall burning rates are obtained with a wider range of the fuel porosity $(\varepsilon=0.6-0.9)$ at $p=0.1$ $\mathrm{MPa}$, and it is found that the overall burning rates vary from $1.4 \mathrm{~mm} / \mathrm{s}$ to $2 \mathrm{~mm} / \mathrm{s}$, depending on the fuel porosity. The maximum overall burning rate is measured with $\varepsilon=0.8$. The reason for the trend of the overall burning rate obtained for the fuel porosity is because, as mentioned, the combustion follows the fuel porosity as shown in Fig. 2. This increasing or decreasing trends of the burning rate versus the fuel porosity at $p=0.1 \mathrm{MPa}$, across the stoichiometric condition are essentially similar to the trend of the laminar burning velocity of premixed-gas mixture versus the equivalence ratio. Looking at the result at $p=0.1 \mathrm{MPa}$, it is, therefore, expected that the overall burning rate is influenced by the (premixedlike) surface flame. However, the experiments in a wide range of the fuel porosity are limited only at $p=0.1 \mathrm{MPa}$, further experiments with various fuel porosity under high pressure environments are needed to support this expectation.

Figure 7 (B) summarizes the overall burning rate $\left(V_{f}\right)$ versus pressure $(p)$ with the fuel porosity $\varepsilon=0.65 \pm 0.05$ and $\varepsilon=0.75 \pm 0.05$. The logarithmic display for both the axes is adopted. It is seen that the overall burning rates span from 1 $\mathrm{mm} / \mathrm{s}$ to $3.2 \mathrm{~mm} / \mathrm{s}$ under the conditions studied, and that the overall burning rate increases as pressure increases and has pressure dependency. This increasing trend of the burning rate with the increase in pressure is qualitatively consistent with one found in the conventional AP-based propellant (Sutton and Biblarz, 2017).

As the results mentioned above, we could successfully obtain those fundamental burning characteristics of the tested specimen, such as the pressure dependency and the fuel porosity on the steadiness, the burning behavior, and the overall burning rate, which the data has been missed since the previous work by Nagata et al. (1997). In this work, it made us 
possible to develop the simple experimental setup to investigate those burning characteristics by adopting hydrogen peroxide (not LOX). For this reason, adopting hydrogen peroxide is suitable to employ as the liquid oxidizer as a first place for the deep understandings of the well-mixing specimen.

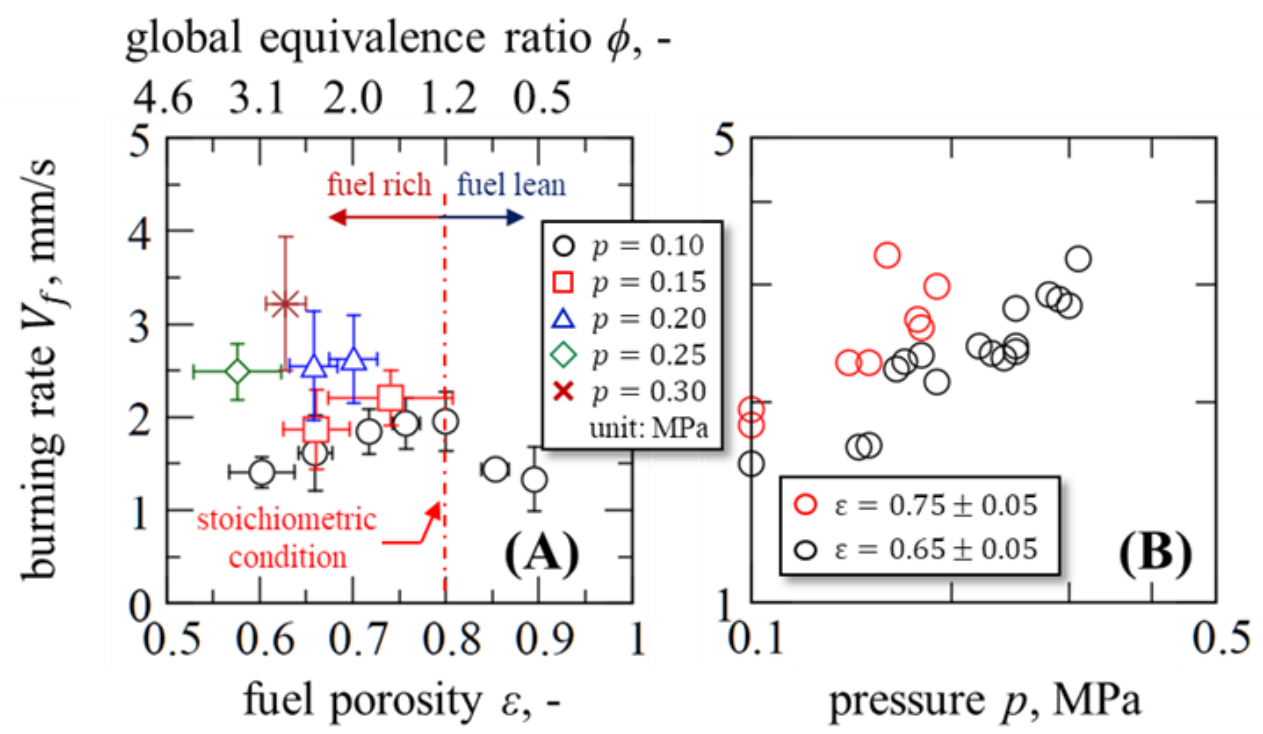

Fig. 7 (A) Overall burning rate vs fuel porosity under various pressure ( $p=0.1 \mathrm{MPa}, 0.15 \mathrm{MPa}, 0.2 \mathrm{MPa}, 0.25 \mathrm{MPa}, 0.3$ $\mathrm{MPa}$ ) and (B) burning rate vs pressure with $\varepsilon=0.65 \pm 0.05$ and $0.75 \pm 0.05$

\subsection{Effect of pressure on thermal structure}

Assuming that a dominant heat flux from the flames to the specimen is thermal conduction through the gas-phase layer between the surface flame and the top surface of the specimen, a thermal structure (e.g., profiles of temperature, temperature gradient, and top surface temperature) is critical to determine the burning rate and can be an evidence to explain the trend shown in Fig. 7. In the conventional AP-based propellant, many research groups have made a great effort to examine the thermal structure by temperature measurement with a fine thermocouple (Sabadell et al., 1965; Aoki et al., 1982; Ishihara et al., 2000).

Apart from the experiments above, additional experiments for temperature measurements are carried out. Fig. 8 shows results of the temperature measurement by the R-type TC embedded into the specimen, for $p=0.1 \mathrm{MPa}, 0.17 \mathrm{MPa}$, and $0.24 \mathrm{MPa}$ in absolute with a constant fuel porosity (around $\varepsilon=0.7$ ). Since we confirmed that the steady burning is observed for all the cases, the time history of temperature profile obtained are converted into profiles of the $x$ coordinate versus temperature by multiplying elapsed time by the overall burning rate. The overall burning rates $V_{f}$ obtained for each condition are denoted on the figure. $x=0$ is adjusted to the top surface temperature of the specimen, and the definition of the top surface temperature will be denoted hereafter. All the axes are adjusted to the same value for better visibility. As it is seen in Fig. 8 (A), the TC passes certain distance around $410 \mathrm{~K}$, and then temperature increases monotonically after the distance to maximum temperature about $1450 \mathrm{~K}(p=0.1 \mathrm{MPa}), 1550 \mathrm{~K}(p=0.17 \mathrm{MPa})$, and $1600 \mathrm{~K}(p=0.24$ $\mathrm{MPa})$, respectively. The temperature profiles finally converge to the maximum temperature beyond the distance of $1 \mathrm{~mm}$ from the location of the top surface temperature. It is suggested that a reaction surface, which may act as "main" heating source to promote the regression of the specimen, namely, the surface flame, is located within the distance of $1 \mathrm{~mm}$. However, it would be hard to precisely identify the exact thermal structure (i.e., a gas-phase (the surface flame) layer and a solid-phase layer)

Then, we converted the temperature profiles into profiles of temperature gradients $(d T / d x)$ to know the exact thermal structure. The temperature gradients are present in Fig. 8 (B) for the same conditions, and an enlarged view of Fig. 8 (B) is shown in Fig. $8(\mathrm{C})$ for better visibility. In (B), it is seen that the peak value increases from $13 \times 10^{5} \mathrm{~K} / \mathrm{m}(p=0.1 \mathrm{MPa})$ up to $17 \times 10^{5} \mathrm{~K} / \mathrm{m}(p=0.24 \mathrm{MPa})$ as pressure increases. With careful observation of the temperature gradients, four regions can be identified: (1) a region where the temperature gradients are almost the constant under about $500 \mathrm{~K}$ (bluecolored region). (2) a region where the temperature gradients increase sharply between about $500 \mathrm{~K}$ and a point below from $x=0$ (green-colored region), (3) a region where the temperature gradients slightly decrease or be flat (gray-colored 
region), and (4) a region where the temperature gradients increase sharply again (red-colored region).

In the region (1), vaporization of hydrogen peroxide occurs ( $410 \mathrm{~K}, p=0.1 \mathrm{MPa}$ in absolute), and the heat is absorbed continuously to vaporize hydrogen peroxide. The reason why there is the certain distance around the vaporization point may be because the boiling hydrogen peroxide is pushed upward through capillary effect. In this region, it is expected that input heat, which affects the temperature gradients, is a constant net heat of thermal conduction transferred from upper side of the specimen and the heat adsorption by the latent heat of the vaporization is constant, so that the constant temperature gradients are obtained in the region (1). Then, it is considered that the profiles of region (2) may be attributed to peculiar reaction phenomenon of hydrogen peroxide utilization, namely, an exothermic reaction processes. Hydrogen peroxide is vaporized around $410 \mathrm{~K}$ ( $p=0.1 \mathrm{MPa}$ in absolute) into vapor, and the vapor will then decompose into water vapor and molecular oxygen while releasing a lot of heat by the exothermic reaction process until around $760 \mathrm{~K}$, as reported in a literature (Giguère and Liu, 1957). Based on the fact, it is considered that this region receives the thermal conduction and the heat by the exothermic reaction process. Therefore, the temperature gradients increase sharply accordingly. In the region (3), the reason for the decrease or flat trend of the temperature gradients may be attributed to the thermal pyrolysis process of the PE foam. In the region (4), the temperature gradients are sharply increased. Thus, the region (4) is indicated as the gas-phase layer. Note that the temperature profile is quite smooth, and the abrupt change in the temperature profile around the surface cannot be identified as shown in Fig. 8 (A). Nevertheless, spatial derivation of the temperature profile (see Fig. 8 (B)) clearly shows that there is obvious change in the thermal structure around the top surface. In addition, there is always minimum value of the temperature gradient in the regions (3) and (4) before the sharp increase as shown in Fig. 8 (C). Based on these observation facts, we would consider that the top surface temperature of the specimen can be defined as the point where the temperature gradient is minimum around the regions (3) and (4). The top surface temperature obtained in the experiments is then denoted on the enlarged view.

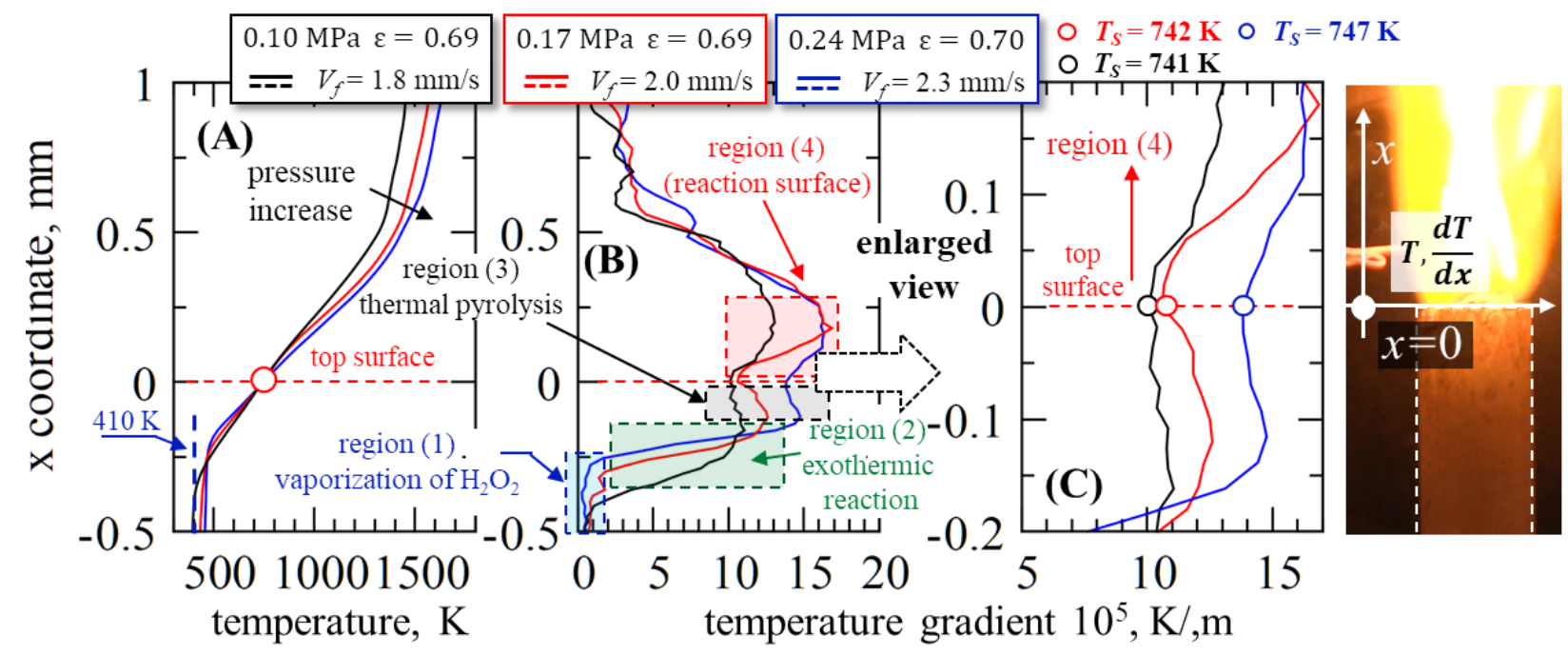

Fig. 8 Profiles of (A) temperature and (B) temperature gradient obtained from direct temperature measurement trials using R-type thermocouple during the entire burning event for $p=0.1 \mathrm{MPa}, 0.17 \mathrm{MPa}$, and $0.24 \mathrm{MPa}$ with a constant fuel porosity $\varepsilon=0.7$, and (C) enlarged view of regions (3) and (4) with values of top surface temperature

As mentioned, the dominant heat flux is determined by the temperature gradient near the top surface in the gas-phase layer. It seems that the peak value of the temperature gradients increases as pressure increases. Hence, the heat flux exposed onto the top surface increases, leading to the increasing trend of the overall burning rate as shown in Fig. 7 (B). Additionally, After the TC passes at $x=0.5 \mathrm{~mm}$, the temperature gradients (in the outer flame) are much lower than that of the surface flame. Therefore, it is found that the outer flame does not affect the overall burning rates, rather, the dominant heating source shall be the surface flame. This fact supports our expectation mentioned in this section.

\subsection{Effect of pressure on top surface temperature and global activation energy}

In order to obtain a global activation energy of the present specimen, the top surface temperature and the burning rate 
obtained in temperature measurements are used with the Arrhenius pyrolysis law (DeLuca et al., 1999) as follows.

$$
V_{f} \propto \exp \left[-\frac{E_{S}}{R T_{S}}\right]
$$

where $E_{S}[\mathrm{~J} / \mathrm{mol}]$ is the global activation energy at the top surface temperature, which is characteristic of the family of chemicals that the propellant belongs to (Beckstead et al., 2007), $R[\mathrm{~J} /(\mathrm{mol} \cdot \mathrm{K})]$ is the universal gas constant, and $T_{S}[\mathrm{~K}]$ is the top surface temperature of the specimen, respectively. Many research groups (Sabadell et al., 1965; Aoki et al., 1982; DeLuca et al., 1999; Ishihara et al., 2000; Servieri et al., 2007) have ever tried to obtain the global activatton energy of polymetic fuels used for the AP-based propellant. In addition, it is reported that the top surface temperature is not consistent with pressure, type of solid fuel, and heating rate (Sabadell et al., 1965; Ishihara et al., 2002). For this reason, the pressure dependency on the top surface temperature and the global activation energy at the top surface temperature in the present specimen are sought to obtain further burning characteristics of the present specimen.

Figure 9 (A) displays the pressure dependency on the top surface temperature and the overall burning rate. The horizontal axis, the vertical axis, and the second vertical axis are denoted as pressure, the top surface temperature, and the overall burning rate measured in the temperature measurement at the same time. The dashed lines are fitted by the least squared method. It is found that the top surface temperature is in a range between $740 \mathrm{~K}(p=0.1 \mathrm{MPa})$ and $750 \mathrm{~K}$ $(p=0.24 \mathrm{MPa})$, and the overall burning rates are increased with the same trend in Fig. 7 (B). As expected, the top surface temperature is not consistent with pressure and increases as pressure increases. In addition, it is seen that the top surface temperature increases with the increase in the overall burning rate. These trends were obtained in the previous works (DeLuca et al., 1999; Servieri et al., 2007)
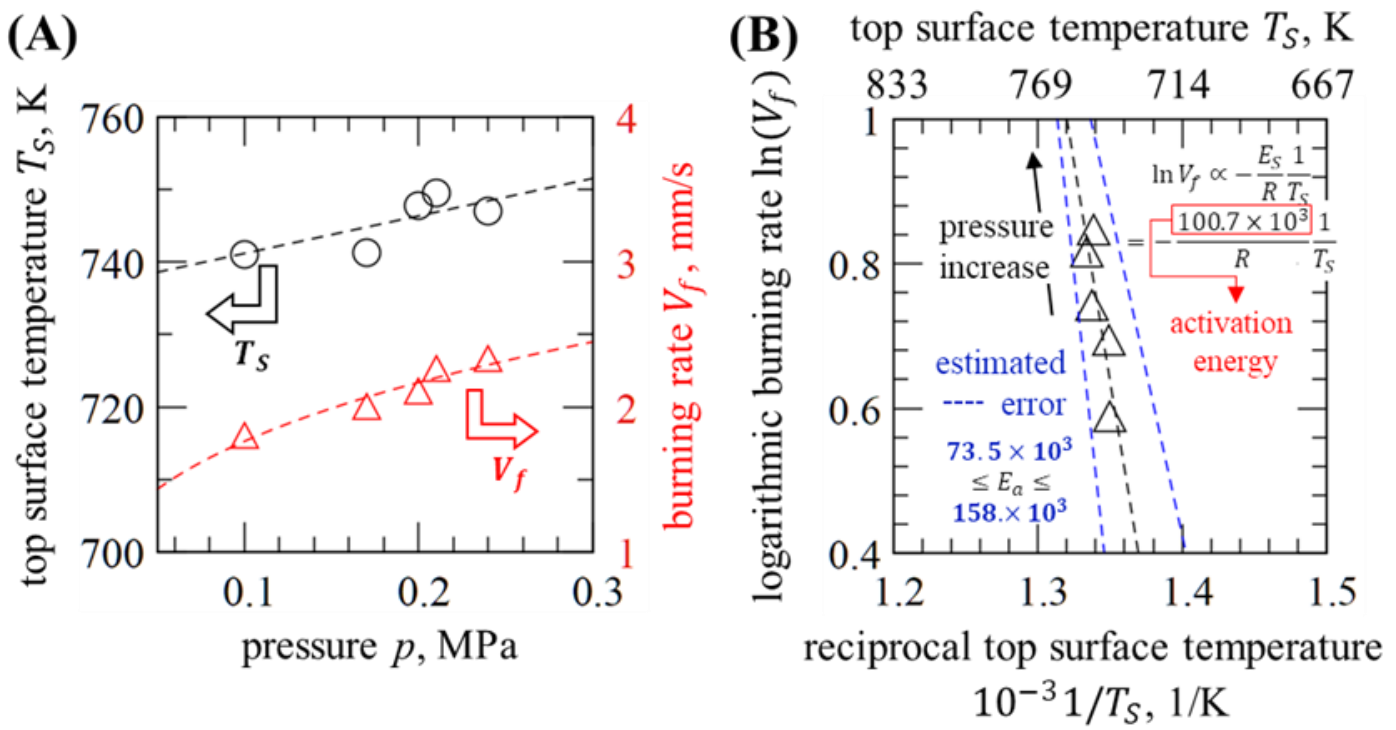

Fig. 9 (A) Top surface temperature (burning surface temperature) obtained from thermocouple embedded into specimen and overall burning rate as a function of pressure and (B) relationship between log natural overall burning rate (ln $\left(V_{f}\right)$ ) and reciprocal burning surface temperature $\left(1 / T_{S}\right)$, together with global activation energy $\left(E_{S}\right)$ at top surface with the estimated errors

By taking a logarithmic expression of Eq. (5), the global activation energy at the top surface temperature can be derived from a gradient of the log natural burning rate $V_{f}\left(\ln \left(V_{f}\right)\right)$ and a reciprocal top surface temperature $\left(1 / T_{S}\right)$. Fig. 9 (B) shows the relationship between $\ln \left(V_{f}\right)$ and $\left(10^{-3} 1 / T_{S}\right)$. The horizontal axis, the vertical axis, and the second horizontal axis are shown as the reciprocal top surface temperature $\left(10^{-3} 1 / T_{S}\right)$, the log natural burning rate $\left(\ln \left(V_{f}\right)\right)$, and the corresponding top surface temperature $\left(T_{S}\right)$. The obtained data are then fitted with a relation denoted on the figure. This result reveals that the global activation energy at the top surface temperature is determined as $100.7 \mathrm{~kJ} / \mathrm{mol}$. It is reported that the conventional values of the global activation energies of the polymeric solid fuels, such as PMMA and HTPB, were in a range between $70 \mathrm{~kJ} / \mathrm{mol}$ and $200 \mathrm{~kJ} / \mathrm{mol}$ (Ishihara et al., 2005; Krishnamurthy et al., 1973; Hertzberg et al., 1991). Especially for the PE foam, an article has revealed that the global activation energy was derived as $119 \mathrm{~kJ} / \mathrm{mol}$ 
(Park et al., 2020). Therefore, the obtained global activation energy exhibits the same order as the polymeric fuels and fairly good agreement with the previous work by Park et al. (2020). Additionally, the global activation energy obtained in this work is not pressure dependent and is rather determined as an eigenvalue of the present system (DeLuca et al., 1999; Servieri et al., 2007). On the other hand, as it is seen in Fig. 7 (A) and Fig. 9 (A), the overall burning rate varies about $1 \mathrm{~mm} / \mathrm{s}$ at maximum $(\varepsilon=0.63, p=0.3 \mathrm{MPa})$, and the top surface temperature varies about $5 \mathrm{~K}$ from the dashed line at maximum $(p=0.17 \mathrm{MPa})$ in the present work. Taking into the account the fact, the global activation energy shall vary in a range between $75.5 \mathrm{~kJ} / \mathrm{mol}$ and $153.7 \mathrm{~kJ} / \mathrm{mol}$ within the measurements conducted in this present work.

\section{Concluding remarks}

In this work, the comprehensive experimental studies to investigate the fundamental burning characteristics (e.g., pressure and the fuel porosity dependency on burning behavior, the overall burning rate, and steadiness) of the replaced tested specimen consisting of the polyethylene foam (PE foam) soaked in $100 \mathrm{wt} \%$ hydrogen peroxide, as referred to our previous work by Matsugi et al. (2021), were made. The experiments at the initial pressure range from $p=0.1$ to $p=0.35$ $\mathrm{MPa}$ in absolute and the fuel porosity range from $\varepsilon=0.6$ to $\varepsilon=0.9$ were carried out in the large volume chamber which was newly introduced in our previous work. Additionally, the temperature measurements using the R-type thermocouple were then made in order to investigate the thermal structure of the burning specimen for deep understandings of the burning process. The findings revealed that the (steady) overall burning rates span from $1.0 \mathrm{~mm} / \mathrm{s}$ to $3.2 \mathrm{~mm} / \mathrm{s}$ and increase when the fuel porosity approaches to the stoichiometric condition (the fuel porosity $\varepsilon=0.8$ ) and as pressure increases. The direct observation of the burning event revealed that the burning behavior in the present specimen was considered as the end burning mode at which the top surface moves downward. The results of the temperature measurements showed that the peak values of the temperature gradients in the gas-phase layer increased as pressure increases and were located very close to the top surface at which the main heating source (the surface flame) is expected to be located. It was lastly found that the top surface temperature (burning surface temperature) had the pressure dependency, and the global activation energy at the top surface temperature obtained in this work was $100.7 \mathrm{~kJ} / \mathrm{mol}$, and this value is quantitatively good agreement with the previous work. By the replaced tested specimen and the newly introduced experimental setup stated in this work, it made us possible to examine not only the fundamental burning characteristics, but also the global activation energy and top surface temperature of the tested specimen.

\section{Acknowledgements}

Technical assistance for the discussion by Dr. Xiaoyu Ju and experimental support by the former student, Mr. Tatsuki Kojima from Toyohashi University of Technology, are greatly appreciated.

\section{References}

Aggarwal, R., Patel, I. K. and Sharma, P. B., Green Propellant: A Study, International Journal of Latest Trends in Engineering and Technology, vol. 6, (2015), pp. 83-87.

Aoki, I. and Kubota, N., Combustion wave structures of high- and low-energy double-base propellants, AIAA, Journal of American Institute of Aeronautics and Astronautics, vol. 20, (1982), AIAA80-1165R.

Aziz, A., Mamat, R., Ali, W. K. W. and Perang, M. R. M., Review on typical ingredients for ammonium perchlorate based solid propellant, Applied Mechanics and Materials, vol. 773-774, (2015), pp. 470-475.

Beckstead, M. W., Puduppakkam, K., Thakre, P. and Yang, V., Modeling of combustion and ignition of solid-propellant Ingredients, Progress in Energy and Combustion Science, vol. 33, (2007), pp. 497-551.

Chaturvedi, S. and Dave, P. N., "Review on thermal decomposition of ammonium nitrate," Journal of Energetic Materials, (2013), pp. 1-26.

DeLuca, L., Cozzi, F., Germinias, G., Ley, I. and Zenin, A. A., Combustion mechanism of an RDX-based composite propellant, Combustion and Flame, vol. 118, (1999), pp. 248-261.

Essel, J. T., Nelson, A. P., Smilowitz, L. B., Henson, B. F.,Merriman, L. R., Turnbaugh, D., Gray, C.and Shermer, K. B., Investigating the effect of chemical ingredient modifications on the slow cook-off violence of ammonium perchlorate solid propellants on the laboratory scale, Journal of Energitic Materials, vol. 38, (2019), pp. $127-141$. 
Fitzgerald, R. P. and Brewster, M. Q., Flame and surface structure of laminate propellants with coarse and fine ammonium perchlorate, Combustion and Flame, vol. 136, (2004), pp. 313-326.

Giguère, P. A. and Liu I. D., Kinetics of the thermal decomposition of hydrogen peroxide vapor, Canadian Journal of Chemistry, (1957), Number 4.

Hertzberg, M. and Zlochower, I. A., Devolatilization wave structures and temperatures for the pyrolysis of polymethylmethacrylate, ammonium perchlorate, and coal at combustion level heat fluxes, Combustion and Flame, vol. 84, (1991), pp. 15-37.

ImageJ, (online), available from https://imagej.nih.gov/ij/, (accessed on 20 March 2021).

Ishihara, A., Sakai, Y., Konishi, K. and Andoh, E., Measurement of the burning surface temperature in ammonium perchlorate, Proceedings of the Combustion Institute, vol. 28, (2000), pp. 855-862.

Ishihara, A., Sakai, Y. Konishi, K. and Andoh, E., Effect of pressure on the burning surface temperature of a polymeric fuel for a solid propellant, Proceedings of the Combustion Institute, vol. 29, (2002), pp. 2873-2879.

Ishihara, A., Sakai, Y., Konishi, K. and Andoh. E., Correlation between burning surface temperature and regression rate for polymethylmethacrylate, Proceedings of the. Combustion Institute, vol. 30, (2005), pp. 2123-2130.

Krishnamurthy, L. and Williams, F. A., Laminar combustion of polymethylmethacrylate in O2/N2 mixtures, Proceedings of Combustion Institute, vol. 14, (1973), pp. 1151-1164.

Matsugi, D., Matsuoka, T. and Nakamura, Y., Study of successive-burning characteristics of porous combustible soaked in liquid oxidizer, Combustion and Flame, vol. 233, (2021), 111571.

McCrain, L. L. and Roberts, W. L., Measurements of the soot volume field in laminar diffusion flames at elevated pressures, Combustion and Flame, vol. 140, (2005), pp. 60-69.

Nagata, H., Akiba, R., Tanatsugu, N., Kohno, M., Yokota, R. and Kayuta, S., A Preliminary study of the wet towel hybrid rocket motor, Aeronautical and Space Sciences Japan, vol. 45, (1997), pp. 365-370 (in Japanese).

Nagata, H., Ito, M., Maeda, T., Watanabe, M., Uematsu, T., Totani, T. and Kudo, I., Development of CAMUI hybrid rocket to create a market for small rocket experiments, Acta Astronautica. vol. 59, (2006), pp. 253-258.

Park, J. W., Lim, W. J. and You, W. J., Effect of ignition heat source on design fire curve of polyethylene foam in a compartment fire, Case Studies in Thermal Engineering, vol. 22, 100790, (2020).

Sabadell, A. J., Wenograd, J. and Summerfield, M., Measurement of temperature profiles through solid-propellant flames using fine thermocouples, AIAA, Journal of American Institute of Aeronautics and Astronautics, vol. 3, (1965), pp. 1580-1584.

Sabourin, J. L., Risha, G. A., Yetter, R. A., Son, S. F. and Tappan, B. C., Combustion characteristics of nanoaluminum, liquid water, and hydrogen peroxide mixtures, Combustion and Flame, vol. 154, (2008), pp. 587-600.

Servieri. M., Galfetti, L., DeLuca, L, Egorshev, V. Y., Kondrikov, B. N. and Grebenyuk, I. V., Burning Mechanism of a Composite Propellant, the proceedings of 33rd International Annual Conference of ICT: Energetic Materials Ignition, Combustion and Detonation, Karlsruhe, Germany, No. 7, 2002, pp. 1-14.

Shioya, S., Kohga, M. and Naya, T., Burning characteristics of ammonium perchlorate-based composite propellant supplemented with diatomaceous earth, Combustion and Flame, vol. 161, (2014), pp. 620-630.

Sundaram, D.S. and Yang, V., Combustion of micron-sized aluminum particle, liquid water, and hydrogen peroxide mixtures, Combustion and Flame, vol. 161, (2014), pp. 2469-2478.

Sutton, G. P. and Biblarz, O., Rocket propulsion elements, Wiley 9th edition, (2017), pp. 446-496.

Wingborg, N. and Calabro, M., Green solid propellants for launchers, Space Propulsion Conference, (2016), SP2016_3125163.

Zhu, M., Chen, X., Zhou, C., Xu, J., Musa, O. and Xiang, H., Experimental and numerical investigations on the decomposition and combustion characteristics of composite propellant with $\mathrm{Mg} / \mathrm{Al}$ particles additives, Applied Thermal Engineering, vol. 111, (2017), pp. 863-875. 